\title{
Adjunctive Treatment of Gummy Smile Using Botulinum Toxin Type-A (Case Report)
}

\author{
Dolly P. Patel ${ }^{1}$, Sandip A.Thakkar ${ }^{2}$, Jaymin R.Suthar ${ }^{3}$ \\ 1-Professor and head, Dept.of orthodontics, Govt.dental college and hospital, Ahmedabad,Gujarat,India \\ 2, 3-PG part-III., Dept.of orthodontics, Govt.dental college and hospital, Ahmedabad, Gujarat, India
}

\begin{abstract}
A 13 Year old female came for a consultation with chief complaints of protrusive lips and a gummy smile. The clinical examination showed a convex profile, a retrognathic mandible, excessively proclined and extruded maxillary incisors, and skeletal class II malocclusion with bimaxillary dento alveolar protrusion. Treatment was started using conventional mechanics for intrusion and retraction of upper anterior teeth, thereby improving soft tissue profile and correction of gummy smile. But only orthodontic treatment was not enough for correction of excessive gingival display during smile secondary to hyperactive upper labial muscle activity. Thus injection of Botulinum toxin type-A was given to correct gummy smile as an adjunctive treatment. Excellent correction of gummy smile following 2 weeks of Botox injection was achieved without any side effects. The patient received a satisfactory occlusion and an attractive smile.
\end{abstract}

Keywords: Gummy smile, neuromuscular correction, Botox.

\section{Introduction}

Recognizably cosmetic dental treatment dates back more than four millennia. According to Hulsey, "A smile is one of the most effective means by which people convey their emotions." The smile, along with speech, is what most visibly displays the results of orthodontic treatment; therefore, smile esthetics becomes a major goal in orthodontic treatment. Often, the main reason people seek orthodontic treatment is to improve dental esthetics. $^{1}$

Patients and parents on orthodontic treatment demands and appreciates more of aesthetic harmony, so along with functionally efficient and balanced occlusion, good esthetics - dynamic smile has become one of the treatment goals of the orthodontist. ${ }^{2}$ The best orthodontically treated subjects may not be satisfied by the treatment, if soft tissue problem is not corrected. Patient desire to look good not only in a static pose but also during dynamic facial expression.

\section{Gummy Smile}

When an excess of gingiva superior to the maxillary anterior teeth is displayed upon full smile, it is termed a gingival smile. The gingival smile is known by a variety of terms including "gummy smile, high lip line, short upper lip, and full denture smile." Perhaps this variety in terms is indicative of the many different causes of a gummy smile. ${ }^{3}$

The smile itself and the aesthetics of the smile are influenced by 3 components: teeth, gums, and lips. An attractive smile depends on the proper proportion and arrangement of these 3 elements. The upper lip should symmetrically expose up to $3 \mathrm{~mm}$ of the gum and the gum line must follow the contour of the upper lip. The exposure of more than $3 \mathrm{~mm}$ of the gum during the smile is known as gingival or gummy smile. For some patients, gummy smile represents an aesthetic disorder. Hulsey noted that the most attractive smiles were those in which the upper lip rested at the height of the gingival margin of the maxillary incisor.

Gummy smiles range from mild, moderate, and advanced, to severe. Rosemarie Mazzuco et al., classified gummy smile into anterior, posterior, mixed, or asymmetric, based on the excessive contraction of muscles involved. ${ }^{4}$

Goldstein classified the smile line (consisting of the lower edge of the upper lip during the smile) according to the degree of exposure of the teeth and gums into 3 types: high, medium, or low. ${ }^{5}$

Botulinum toxin has been under clinical investigation since the late 1970s for the treatment of several conditions associated with excessive muscle contraction or pain. Botulinum toxin is produced by the anaerobic bacterium Clostridium botulinum. There are 8 different serotypes of botulinum toxin. Type A (BTX-A) is the most potent and the most commonly used clinically. Botox (Allergan, Irvine,Calif) is a purified BTX-A isolated from the fermentation of $C$ botulinum. ${ }^{6}$

It is a stable, sterile, vacuum-dried powder that is diluted with saline solution without preservatives. BTX-A weakens skeletal muscles by cleaving the synaptosome-associated protein SNAP-25, thus blocking the release of acetylcholine from the motoneuron and enabling the repolarization of the postsynaptic term. ${ }^{7}$ 
In this case report, we aimed to introduce the treatment of a woman with a skeletal Class II Angle's class-I malocclusion with extraction of all first premolars followed by Botulinum toxin injection for correction of gummy smile as an adjunctive treatment.

\section{Diagnosis And Etiology}

A 13-year-old Indian Hindu female presented to DEPARTMENT OF ORTHODONTICS AND DENTOFACIAL ORTHOPEDICS, GOVT.DENTAL COLLEGE AND HOSPITAL, AHMEDABAD, and GUJARAT with a chief complaint of protrusive lips and a gummy smile

Pre-treatment photographs showed protrusive upper and lower lips, both of which exceeded the E-line and were strained on closure. (fig-1) No anomaly was seen in the frontal facial photos, except that the patient was overly cautious about smiling naturally. When the patient relaxed, we took another smiling image, which showed a significant gummy smile with more than $4 \mathrm{~mm}$ of gingival exposure in the incisor region. The photographs and pre-treatment study casts exhibited excessively proclined and extruded maxillary incisors, and class-I canine and molar relationships with a deep overbite and overjet and moderate crowding in lower anteriors. The clinical examination suggested incisal and canine guidance without prematurity and shift. The patient had no temporomandibular joint symptoms. No deviation and pain during the border movement of the mandible were discovered. Hyperactive upper lip was found secondary to excessive proclination of upper anteriors.

A pre-treatment cephalogram and a panoramic radiograph were taken. The Cephalometric analysis and the tracing demonstrated a Class II skeletal relationship as a result of the retruded mandible. The A-point was in the normal range (fig 2 and 3) (SNA, 82 degree), and B-point was retruded (SNB, 75 degree). The angle between the maxillary incisors and the S-N plane was 118 degree, and the IMPA was 102 which indicated that skeletal class-II with bimaxillary dento-alveolar protrusion.

\section{Treatment Objectives}

The treatment objectives were to maintain occlusion with a Class I molar relationship and normalize overjet and overbite. Retraction and intrusion of the maxillary anterior teeth were indicated to reduce the exposure of the gingiva and the protrusive profile. Simultaneously, extrusion of the posterior teeth would be limited to prevent a clockwise rotation of the mandible and an increase in lower facial height.

\section{Treatment Alternatives}

Three alternatives were presented to the patient.

1. Combined surgical and orthodontic treatment to harmonize the molar relationship with extraction of premolars in both the maxilla and the mandible. Orthognathic surgery could be used to elevate and retrude the anterior maxillary dentoalveolar part to eliminate the gummy smile and improve the profile.

Advancement genioplasty could be used to camouflage the retruded mandible and improve the profile.

2. Harmonize the molar relationship and retract the anterior teeth by extracting all first premolars. Use TADs to provide absolute anchorage for maximum retraction of the proclined maxillary teeth and maxillary incisor intrusion to eliminate the gummy smile.

3. Extract the first premolars in both the arches, and use J-hook headgear

as anchorage for retraction and intrusion. The disadvantage was that the effect of this treatment depended on the patient's cooperation.

As the patient was in growing stage (Age 13), option of orthognathic surgery for correction of this malocclusion can be put as a last choice. After we had discussed about pros and cons of second option, patient became apprehensive. She considered the improvement from use of implants not worth the additional risk and cost. So we decided to start our treatment by extraction of first pre-molars in both the arches followed by retraction and intrusion of upper anterior teeth using J-Hook head-gear.

Head-gear was given as an anchorage for retraction and intrusion but the co-operation from the patient needed for its use couldn't be achieved. So we decided to start our treatment using conventional mechanics for retraction and intrusion of upper anterior teeth and later on use of Botulinum toxin if gummy smile still persists.

\section{Treatment Progress}

Treatment began with the placement of straight wire brackets (0.022-in slot MBT) on all maxillary and mandibular teeth for levelling and aligning. Nance palatal arch in upper and lingual arch in lower arch for maintaining molars and canines in class-I relationship bilaterally and for anchorage purpose were given. After initial alignment and levelling for 5 months extraction of first premolars were done in both arches. Wire progression in sequence from .016 in NiTi, .016x.022 in HANT, .016x.022 in SS, .017x.025 in HANT done before extractions were performed. 
Separate canine method for retraction was done in upper arch. After achieving canine retraction K-SIR (Kalra Simultaneous Intrusion and Retraction) loop with loops in between upper lateral incisors and canines using .019x.025 in TMA arch wire was given for simultaneous intrusion and retraction of anteriors.

For lower arch en mass retraction method was used.En mass retraction was done by using sliding mechanics with hooks welded on .019x.025 in SS arch wire mesial to canines was done in lower arch. After achieving proper intrusion, finishing was done by using .019x0.25 SS wire for 3 months in both the arches.

It took 18 months for orthodontic correction. Following finishing and settling of occlusion still gummy smile was evident due to hypertonic upper lips secondary to excessive pre-treatment protrusion of upper anterior teeth.

By using conventional mechanics as judiciously as possible for intrusion of anteriors, complete correction of excessive gingival display during smile couldn't achieved. Gingival display during smile was still $3.5 \mathrm{~mm}$ at this point of treatment.(fig-4)

At this stage of treatment we discussed about the possible correction of gummy smile that can be achieved using Botulinm toxin Type-A with patient and her parents. Information about the procedure, its possible benefits, risks, side effects, and the expected duration of the results, was given in detail to the patient and the parents verbally and in writing. Patient was motivated for the use of Botox. Written informed consent was obtained.

Injection of $1.25 \mathrm{U}$ of Botulinm toxin type-A was given on both right and left side at YONSEI POINT. Excellent reduction of gingival display during smile, which was $3.5 \mathrm{~mm}$ at midstage become $0 \mathrm{~mm}$ following 2 weeks of injection.(fig-5,6)

\section{Treatment Results}

The post treatment photographs showed a remarkable improvement in lip profile from the significant retraction of the anterior teeth. (Fig-7) Meanwhile, there was no gummy smile following Botox injection. The post treatment photographs demonstrated Class I canine and molar relationships with normal overbite and overjet. The Cephalometric analysis showed no change in the position of the maxillary molars. (Fig 8\& 9) The incisal edge of the maxillary incisors was retracted by $4 \mathrm{~mm}$. Normal overbite and overjet were achieved.

\section{Table. Cephalometric values}

\begin{tabular}{|lccc|}
\hline Measurement & Standard & Initial & Final \\
\hline SNA angle $\left({ }^{\circ}\right)$ & 82 & $\mathbf{8 2}$ & $\mathbf{8 1}$ \\
\hline SNB angle $\left({ }^{\circ}\right)$ & 80 & $\mathbf{7 5}$ & $\mathbf{7 6}$ \\
\hline ANB angle $\left({ }^{\circ}\right)$ & 2 & $\mathbf{7}$ & $\mathbf{5}$ \\
\hline Wits $(\mathrm{mm})$ & 1 & $\mathbf{3}$ & $\mathbf{2}$ \\
\hline SN-Go-Gn $\left({ }^{\circ}\right)$ & 32 & $\mathbf{3 3}$ & $\mathbf{3 3}$ \\
\hline MP to FH $\left({ }^{\circ}\right)$ & 25 & $\mathbf{2 6}$ & $\mathbf{2 5}$ \\
\hline U1 to NA $\left({ }^{\circ}\right)$ & 22 & $\mathbf{3 5}$ & $\mathbf{2 2}$ \\
\hline U1 to NA $(\mathrm{mm})$ & 4 & $\mathbf{1 0}$ & $\mathbf{6}$ \\
\hline L1 to NB $\left({ }^{\circ}\right)$ & 25 & $\mathbf{3 3}$ & $\mathbf{2 6}$ \\
\hline L1 to NB $(\mathrm{mm})$ & 4 & $\mathbf{1 0}$ & $\mathbf{6}$ \\
\hline L1 to MP $\left({ }^{\circ}\right)$ & 90 & $\mathbf{1 0 2}$ & $\mathbf{9 8}$ \\
\hline U1 to $\mathrm{L} 1\left(^{\circ}\right)$ & 130 & $\mathbf{1 0 5}$ & $\mathbf{1 2 6}$ \\
\hline Pog to NB $(\mathrm{mm})$ & 1 & $\mathbf{- 7}$ & $\mathbf{- 5}$ \\
\hline
\end{tabular}

The final Cephalometric analysis showed that the skeletal discrepancy between the maxilla and the mandible was reduced from pre treatment ANB 7 degree to post treatment ANB 5 degree. The comparison between the pre treatment and post treatment panoramic radiographs showed no apparent root shortening.

Though reduction in gummy smile using botulinum toxin injection is reversible phenomenon, but it's very effective adjunctive treatment approach along with fixed mechanotherapy. After 6 months of Botulinm toxin injection, still some amount of correction was retained.

\section{Discussion}

Gummy smiles can be classified by etiology into soft tissue, dentoalveolar, and skeletal types. The skeletal type is caused by excessive vertical maxillary growth and is found in patients with long-face syndrome. ${ }^{8-9}$ Orthognathic surgery is generally required to treat this problem. However, in some dentoalveolar cases, orthognathic surgery could produce an unfavourable result. ${ }^{10}$ For the patient whose gummy smile is derived from protrusion and extrusion of the maxillary anterior dentoalveolar complex, decreased anterior 
dentoalveolar height after surgery might result in a low smile. These considerations, plus the age factor of the patient (13 yrs), suggested that orthognathic surgery was inappropriate for eliminating the gummy smile in our patient. As a result, orthognathic surgery was abandoned after careful consideration.

For this patient, the convex profile and gummy smile were both caused by excessive protrusion of the maxillary anterior dentoalveolar complex. Thus, maximum retraction of the maxillary anterior teeth was needed. As the patient was not ready for any surgical procedure including use of TADs, conventional anchorage means were used for retraction and intrusion of anterior teeth. After retraction and intrusion by means of simple conventional mechanics still excessive gingival display during smile was evident because of hypertonic upper lips.

Thus for neuro-muscular correction in cases with hypertonic upper labial muscles use of Botulinum toxin cannot be ignored. Muscles of facial expression responsible for upper lip elevation and lateral retraction upon smiling are LLSAN, LLS, Zm, zygomaticus major (ZM), risorius, and, to a lesser degree, the depressor septi nasi muscle. All of these muscles interact with the orbicularis oris muscle in the production of a smile.

The mechanism involved is well described in 2 cadaver studies, by Rubin et $\mathrm{al}^{11}$ and another by Pessa ${ }^{12}$. Both investigations evaluated the origin of the nasolabial fold. Rubin et al concluded that the LLS, the ZM, and the superior fibers of the buccinator muscles under the nasolabial fold are responsible for the production of a full smile. Pessa indicated that the LLSAN was responsible for the formation of the medial portion of the fold and minimally responsible for the elevation of the upper lip and smile formation. He also found that the $\mathrm{ZM}$ and the $\mathrm{Zm}$ muscles are primarily responsible for the production of the smile.

\section{BTX-A, the smile, and esthetics}

BTX-A has been under clinical investigation since the late 1970s for the treatment of several conditions associated with excessive muscle contraction. ${ }^{13}$

Produced by the anaerobic bacterium $C$ botulinum, there are 7 serotypes of BTX. BTX-A is the most potent and the most commonly used clinically type. ${ }^{14}$ Botox is a purified BTX-A isolated from the fermentation of $C$ botulinum. It is a stable, sterile, vacuum-dried powder that is diluted with saline solution without preservatives. BTX-A blocks neuromuscular transmission by binding to acceptor sites on motor or sympathetic nerve terminals, thus inhibiting the release of acetylcholine.

This inhibition occurs as the neurotoxin cleaves SNAP- 25, a protein integral to the successful docking and release of acetylcholine from vesicles in nerve endings. ${ }^{15}$ When injected intramuscularly at therapeutic doses, BTX-A produces partial chemical denervation of the muscle, resulting in localized reduction in muscle activity. Botox has been approved by the Food and Drug Administration as a safe and effective therapy for blepharospasm, strabismus, cervical dystonia, and hemifacial spasm since 1989; in 2002, it received approval for the treatment of glabellar lines associated with corrugator and procerus muscle activity, and, in 2004, approval was obtained for the treatment of primary axillary hyperhidrosis. The National Institutes of Health Consensus Conference of 1990 also included it as a safe and effective therapy for other nonlabeled uses. The use of BTX-A for many facial cosmetic procedures has been described extensively in the literature. ${ }^{16}$

After injecting 1.25U Botulinm toxin on both the side, reduction in excessive gingival display was noticed with maximum effect following 2 weeks. Gingival display gradually increased from 2 weeks post injection through 24 weeks, but, at 24 weeks, average gingival display still had not returned to baseline values.

i. 


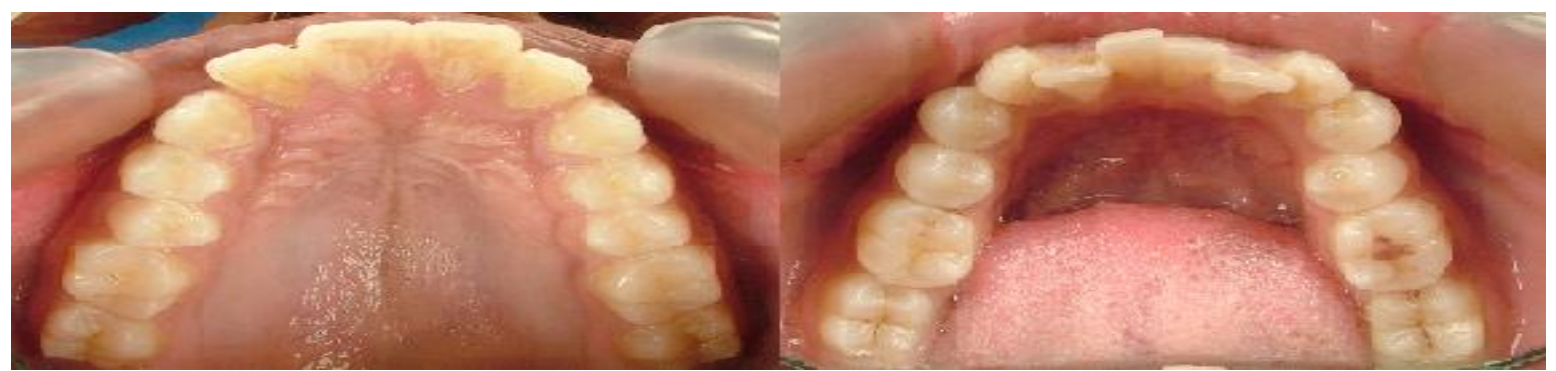

FIG-1 PRE-TREATMENT PHOTOGRAPHS

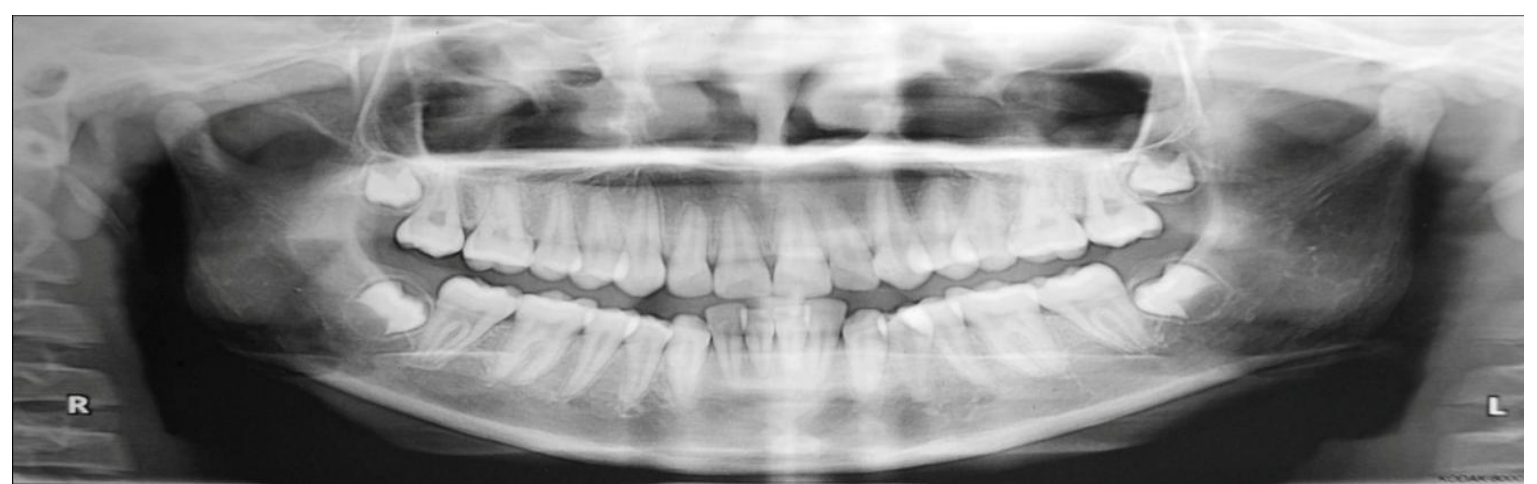

FIG-2 PRE-TREATMENT PANORAMIC RADIOGRAPH

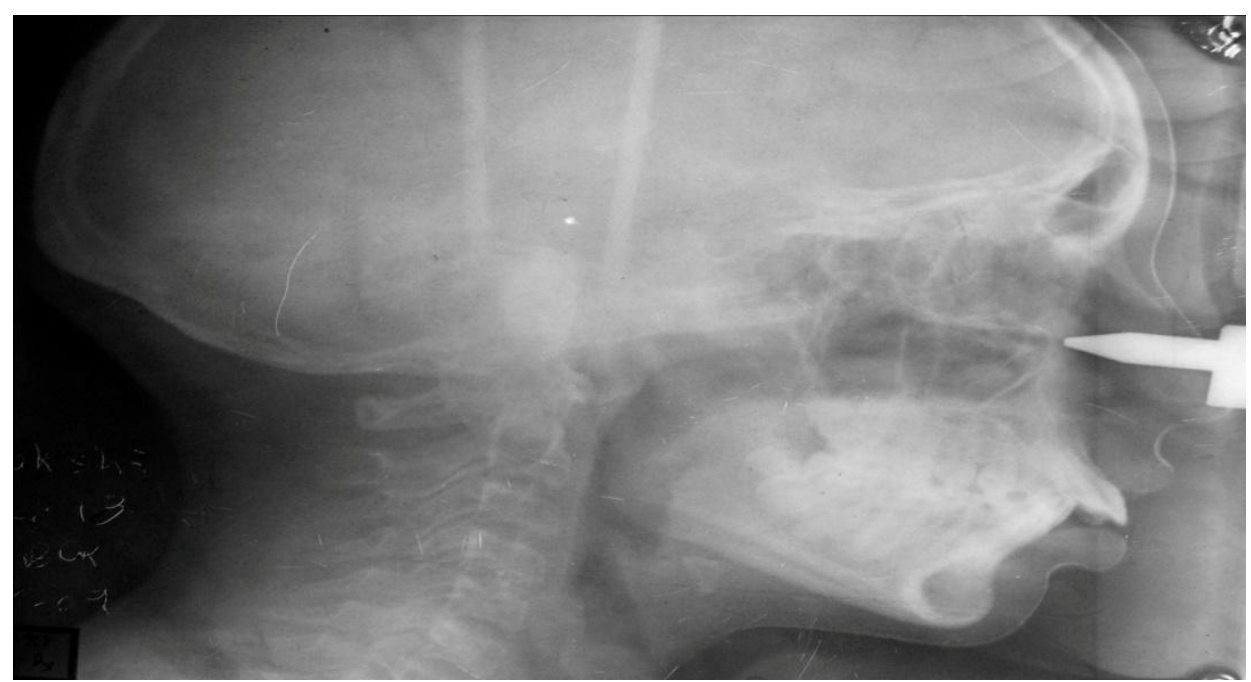

FIG-3 PRE-TREATMENT LATERAL CEPHALOGRAM

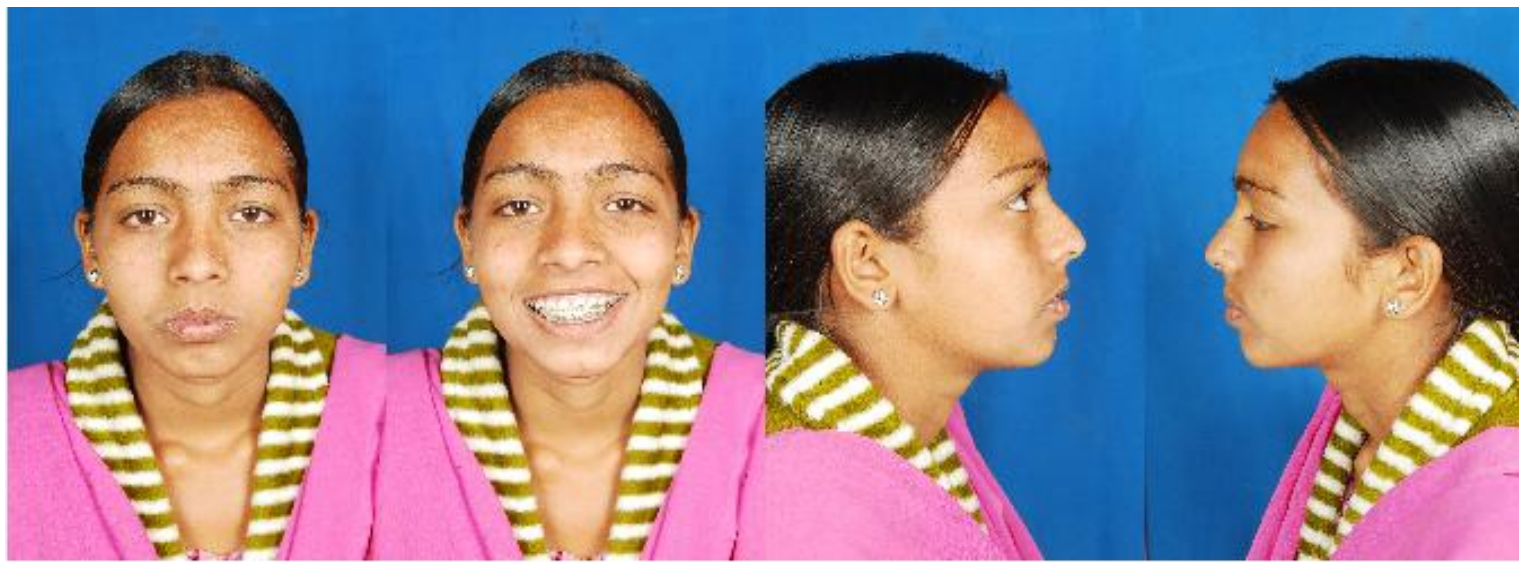




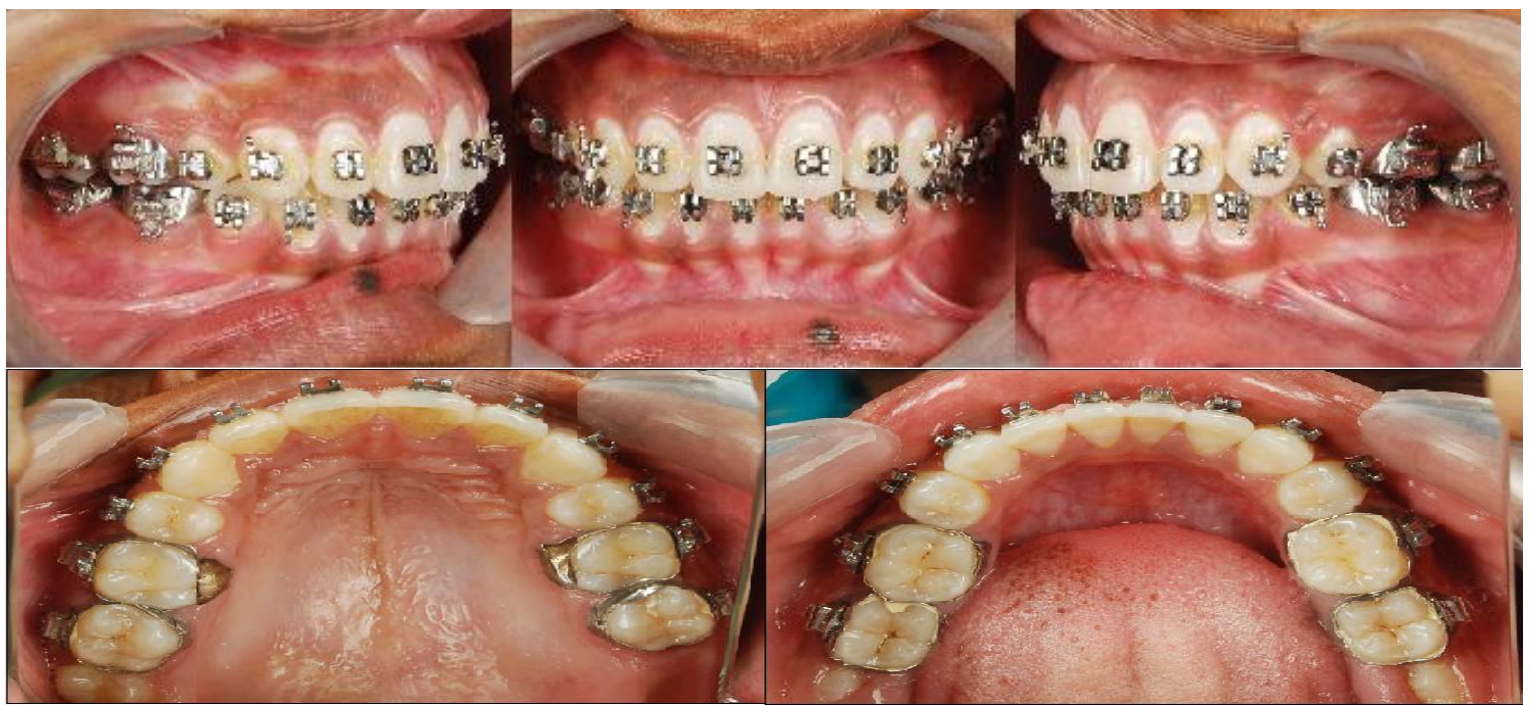

FIG-4 MID-TREATMENT PHOTOGRAPHS

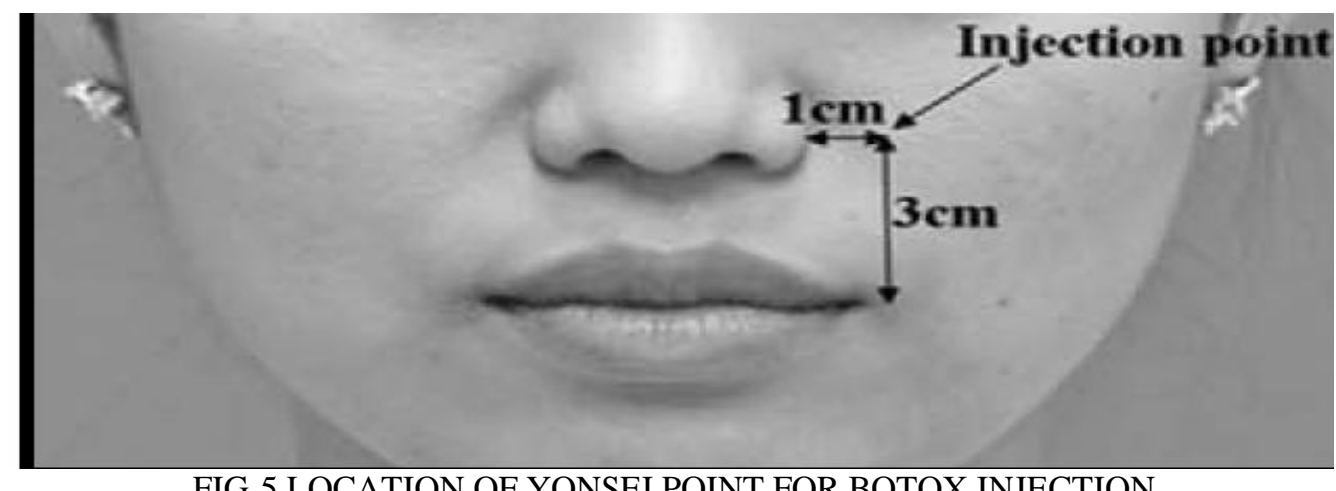

FIG-5 LOCATION OF YONSEI POINT FOR BOTOX INJECTION

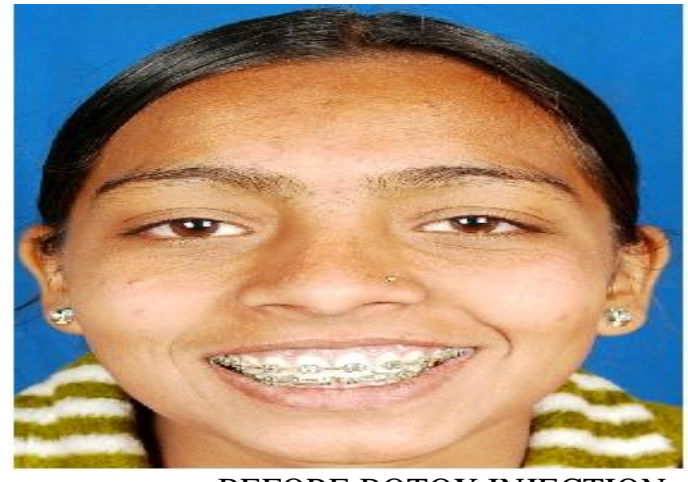

BEFORE BOTOX INJECTION

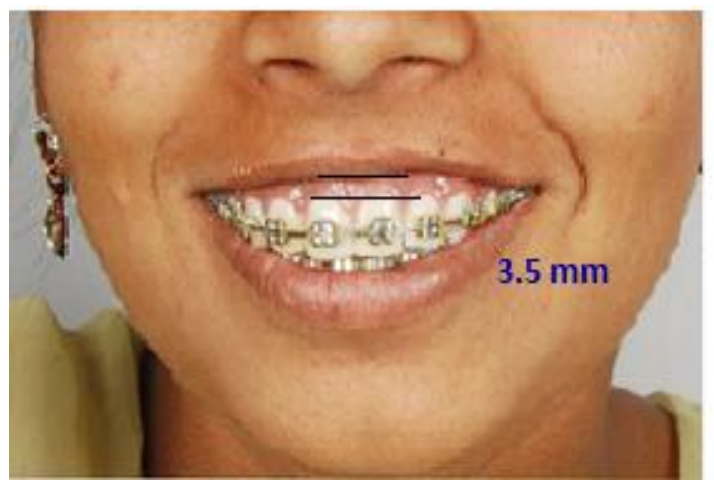

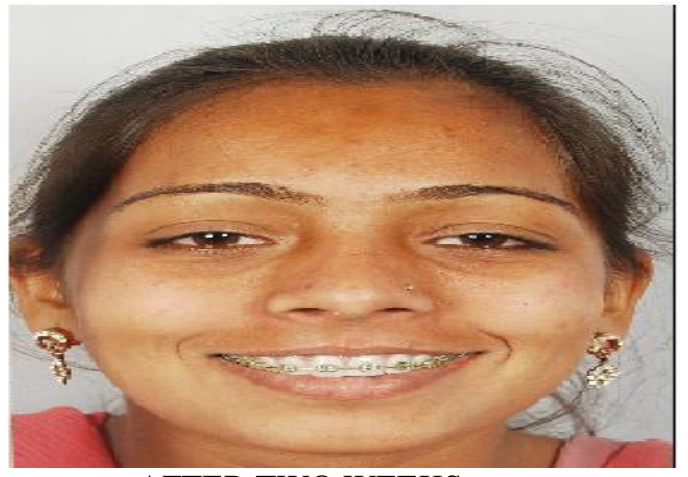

AFTER TWO WEEKS

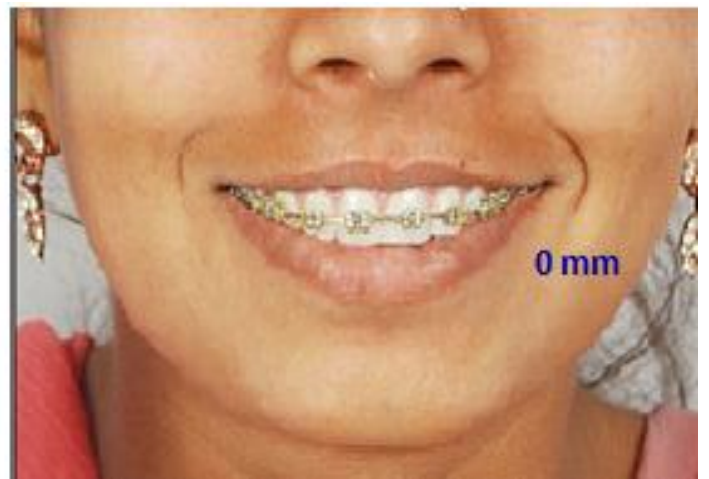

FIG-6 CORRECTION AFTER BOTOX INJECTION 


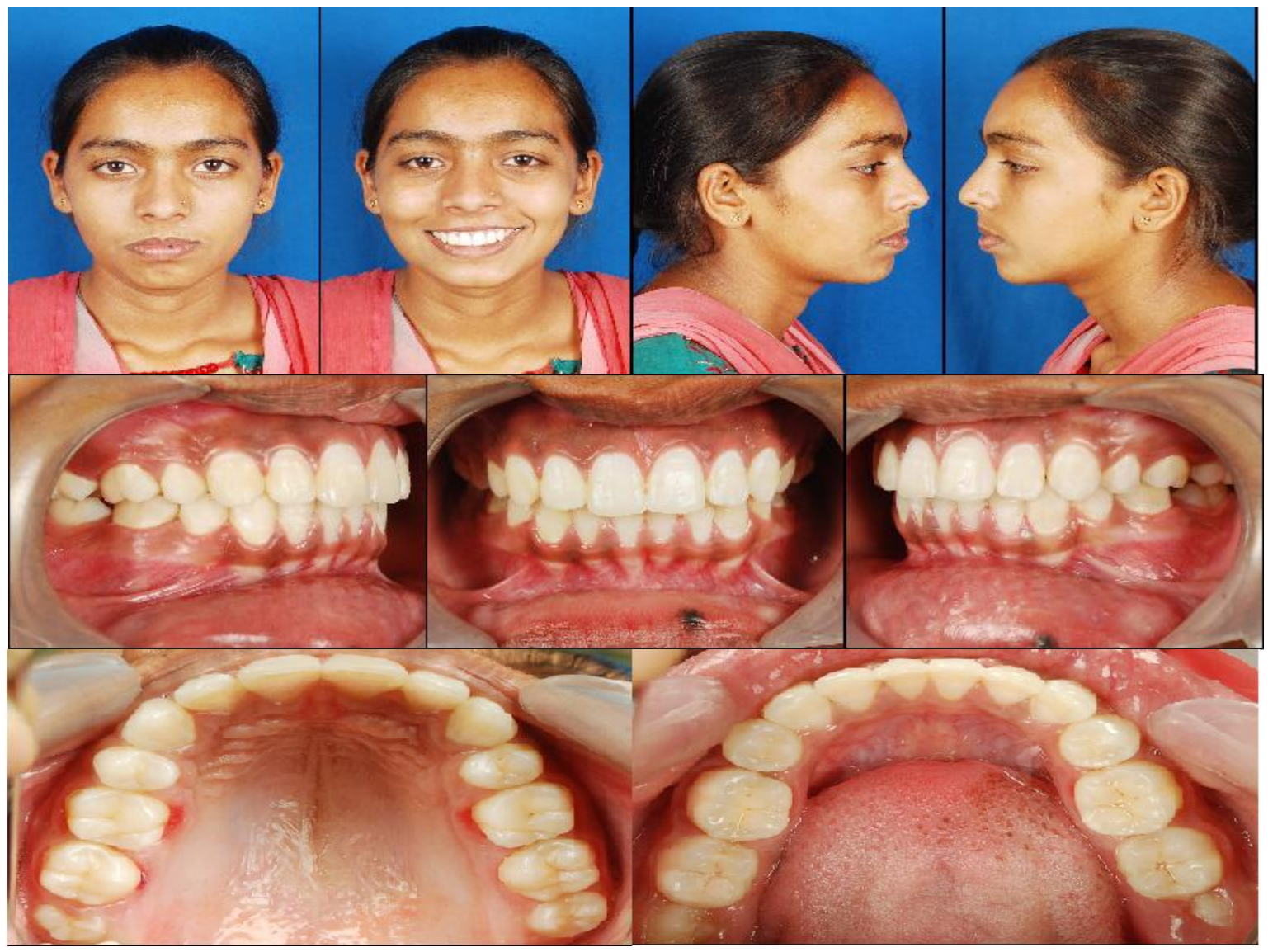

FIG-7 POST TREATMENT PHOTOGRAPHS

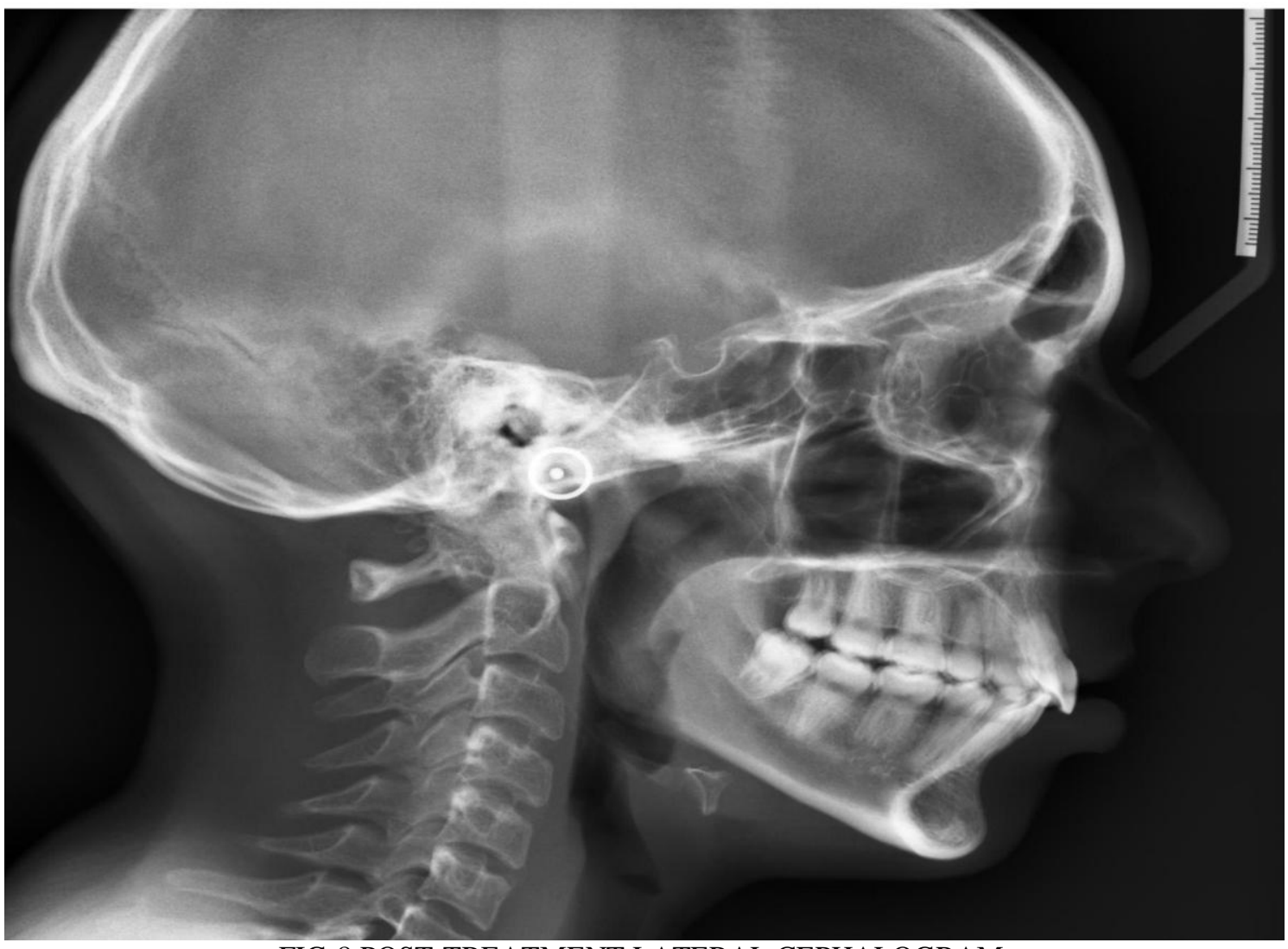

FIG-8 POST-TREATMENT LATERAL CEPHALOGRAM 


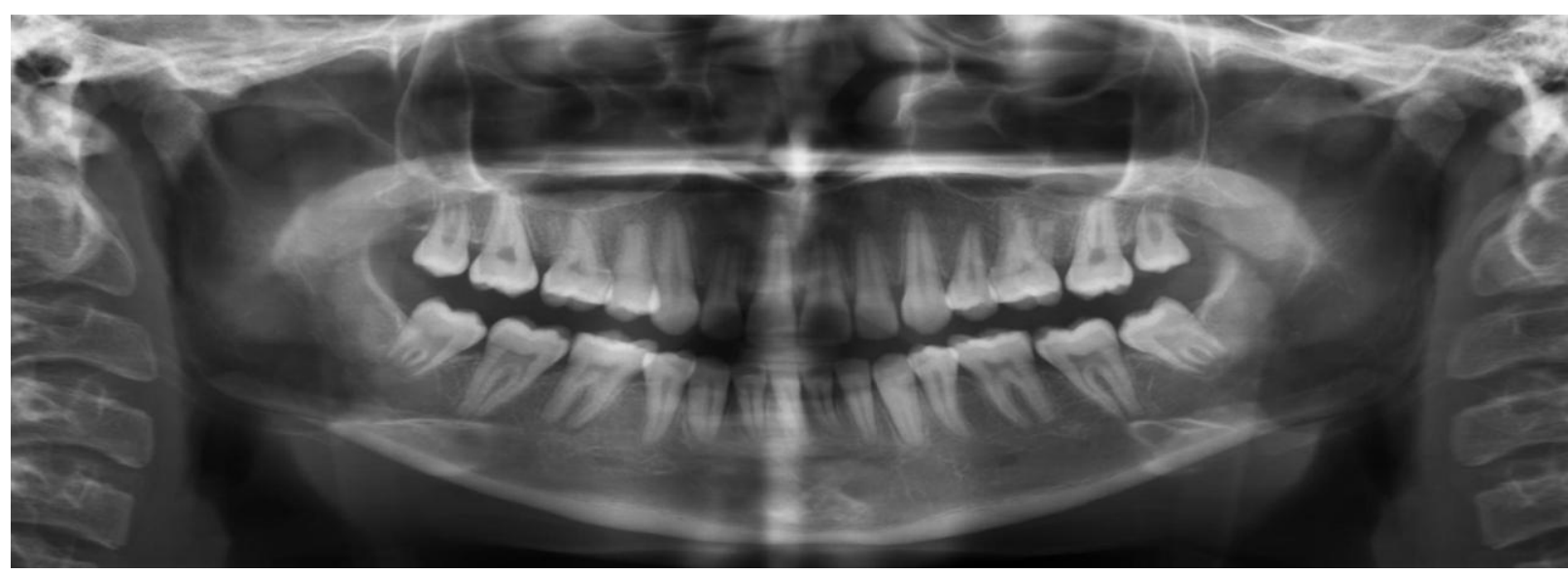

FIG-9 POST-TREATMENT PANORAMIC RADIOGRAPH

\section{Conclusion}

The value of an attractive smile is undeniable. A smile is considered the universal friendly greeting in all cultures. An attractive smile in modern society is often considered an asset in interviews, work settings, social interactions, and even the quest to attract a mate. ${ }^{17} \mathrm{~A}$ pleasing smile involves a harmonious relationship among the teeth, the gingival scaffold, and the lip framework. With the help of orthodontic treatment changes can be made in teeth alignment, and positioning. However orthodontic treatment alone can do little in achieving harmonious smile completely in cases with hyperactivity of lip muscles. These cases may greatly benefited by use of botox.

Thus simply by injecting Botulinm toxin one can reduce excessive gingival display by neuro-muscular correction of hyperactive upper labial muscle activity as an adjunctive treatment along with fixed appliance without any side-effects.

\section{Referances}

[1] Hulsey CM. An esthetic evaluation of lip-teeth relationships present in the smile. Am J Orthod. Dentofacial Orthop.1970; 57 (2): $132-144$.

[2] Ker AJ, Chan R, Fields HW, Beck M, Rosenstiel S.Esthetics and smile characteristics from the layperson's perspective: a compu terbased survey study. J Am Dent Assoc. 2008; 139(10):1318-1327.

[3] Peck S, Peck L, Kataja M. The gingival smile line. Angle Orthod. 1992; 62(2):91-100.

[4] Mazzuco and Hexsel: Gummy smile and botulinum toxin: A new approach based on the gingival exposure area. J AM Acad Dermatol, vol 63, no 6, 1042-1051.

[5] Goldstein RE. Estética em Odontologia. Rio de Janeiro: Ed. Guanabara Koogan; 1980.

[6] Textbook of microbiology, seventh edition-2005,reprint, Ananthnarayan and paniker.

[7] G. W. C. Jaspers, J. Pijpe, J. Jansma: The use of botulinum toxin type A in cosmetic facial procedures. Int. J. Oral Maxillofac. Surg. 2011; 40: 127-133.

[8] Proffit WR, Fields HW, Sarver DM. Contemporary orthodontics. St Louis: Mosby Year Book; 2007.

[9] Robbins JW. Differential diagnosis and treatment of excess gingival display. Pract Periodontontics Aesthet Dent 1999;11:265-72. [10]. Silberberg N, Goldstein M, Smidt A. Excessive gingival display—etiology, diagnosis, and treatment modalities. Quintessence Int 2009;40:809-18.

[10] Pessa JE. Improving the acute nasolabial angle and medial nasolabial fold by levator alae muscle resection. Ann Plast Surg 1992;29:23-30.

[11] Rubin LR. The anatomy of a smile: its importance in the treatment of facial paralysis. Plast Reconstr Surg 1974;53:384-7.

[12] Brin MF, Hallett M, Jankovic J. Preface. In: Brin MF, Hallet M, Jankovic J, editors. Scientific and thera peutic aspects Of botulinum toxin. Philadelphia: Lippincott Williams and Wilkins; 2002.p. v-vi.

[13] Benedetto AV. The cosmetic uses of botulinum toxin type A. Int J Dermatol 1999;38:641-55.

[14] Binder WJ, Blitzer A, Brin MF. Treatment of hyperfunctional lines of the face with botulinum toxin A. Dermatol Surg

[15] 1998;24:1198-205.

[16] Clinical use of botulinum toxin. NIH Consens Statement Online 1990;8:1-20.

[17] Maulik c., Nanda r. Dynamic smile analysis in young adults. Am J Ortho Orthop 2007; 132:307-15 